\title{
Intraperitoneal Activation of Coagulation and Fibrinolysis in Patients with Cirrhosis and Ascites
}

\author{
Johannes Thaler ${ }^{1}$ (1) Ton Lisman $^{2}$ Peter Quehenberger ${ }^{3}$ Lena Hell ${ }^{1}$ Philipp Schwabl ${ }^{4,5}$ \\ Bernhard Scheiner ${ }^{4,5}$ Theresa Bucsics ${ }^{4,5}$ Rienk Nieuwland ${ }^{6}$ Cihan Ay ${ }^{1}$ Michael Trauner ${ }^{4,5}$ \\ Ingrid Pabinger ${ }^{1}$ Thomas Reiberger ${ }^{4,5}$ Mattias Mandorfer ${ }^{4,5}$
}

\footnotetext{
1 Division of Haematology and Haemostaseology, Department of Internal Medicine I, Medical University of Vienna, Vienna, Austria

2 Department of Surgery, Surgical Research Laboratory and Section of Hepatobiliary Surgery and Liver Transplantation, University Medical Center Groningen, University of Groningen, Groningen, The Netherlands

${ }^{3}$ Department of Laboratory Medicine, Medical University of Vienna, Vienna, Austria

${ }^{4}$ Division of Gastroenterology and Hepatology, Department of Internal Medicine III, Medical University of Vienna, Vienna, Austria
}

Address for correspondence Mattias Mandorfer, MD, PhD, Division of Gastroenterology and Hepatology, Department of Internal Medicine III, Medical University of Vienna, Waehringer Guertel 18-20, Vienna, 1090, Austria (e-mail: mattias.mandorfer@meduniwien.ac.at).

${ }^{5}$ Vienna Hepatic Hemodynamic Lab, Medical University of Vienna, Vienna, Austria

${ }^{6}$ Laboratory of Experimental Clinical Chemistry, Vesicle Observation Centre, Academic Medical Center, University of Amsterdam, Amsterdam, The Netherlands

\begin{abstract}
Development of ascites is the most common form of decompensation of cirrhosis. We aimed to investigate the coagulation system in ascitic fluid and plasma of patients with cirrhosis. We determined coagulation parameters and performed clotting and fibrinolysis experiments in ascitic fluid and plasma of thoroughly characterized patients with cirrhosis and ascites $(n=25)$ and in plasma of patients with cirrhosis but without ascites $(n=25)$, matched for severity of portal hypertension. We also investigated plasma Ddimer levels in an independent cohort of patients $(n=317)$ with clinically significant portal hypertension (HVPG $\geq 10 \mathrm{mmHg}$ ), grouped according to ascites severity. Ascitic fluid was procoagulant in a clotting assay. The procoagulant potential of ascitic fluid was abolished by depletion of extracellular vesicles from ascitic fluid by filtration or by addition of a tissue factor-neutralizing antibody. Compared with plasma, extracellular vesicle-associated tissue factor activity was high in ascitic fluid, while activities of other coagulation factors were low. The extracellular vesicle-depleted fraction of ascitic fluid induced fibrinolysis, which was prevented by aprotinin, indicating the presence of plasmin in ascitic fluid. Plasma peak thrombin generation and parameters reflecting fibrinolysis were independently associated with the presence of ascites. Finally, plasma D-dimer levels were independently linked to ascites severity in our second cohort

Keywords

- cirrhosis

- ascites

- coagulation

- fibrinolysis comprising 317 patients. In conclusion, coagulation and fibrinolysis become activated in ascites of patients with cirrhosis. While tissue factor-exposing extracellular vesicles in ascitic fluid seem unable to pass the peritoneal membrane, fibrinolytic enzymes get activated in ascitic fluid and may re-enter the systemic circulation and induce systemic fibrinolysis.
\end{abstract}

received

February 5, 2021

accepted after revision

May 18, 2021

published online

May 21, 2021
DOI https://doi.org/ 10.1055/a-1515-9529. ISSN 0340-6245. (c) 2021. The Author(s).

This is an open access article published by Thieme under the terms of the Creative Commons Attribution-NonDerivative-NonCommercial-License, permitting copying and reproduction so long as the original work is given appropriate credit. Contents may not be used for commercial purposes, or adapted, remixed, transformed or built upon. (https://creativecommons.org/ licenses/by-nc-nd/4.0/)

Georg Thieme Verlag KG, Rüdigerstraße 14, 70469 Stuttgart, Germany 


\section{Introduction}

The liver plays a central role in the hemostatic system since it synthesizes and clears most proteins involved in coagulation activation and fibrinolysis. Hemostatic alterations in patients with advanced chronic liver disease (ACLD) typically include reduced levels of most coagulation factors and their respective inhibitors, as well as reduced fibrinolytic enzymes, ${ }^{1-3}$ since they are synthesized by hepatocytes. In contrast, levels of coagulation factor VIII and von Willebrand factor are increased in ACLD patients who also frequently have thrombocytopenia. ${ }^{4,5}$

Patients with ACLD are nowadays considered to have a balanced hemostatic equilibrium. ${ }^{1-3}$ However, when compared with liver-healthy subjects, this equilibrium seems to be less stable and easily tips in one direction, which together with other factors such as portal hypertension and reduced portal venous blood flow velocity commonly leads to bleeding and/or thrombosis. ${ }^{1-3}$ The term "accelerated intravascular coagulation and fibrinolysis" (AICF) was coined to describe laboratory alterations and clinical features like mucosal oozing and prolonged wound bleeding that are observed in a subset of ACLD patients. ${ }^{6}$ However, the patho-

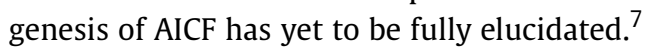

Development of ascites, the most common first decompensating event in patients with $\mathrm{ACLD},{ }^{8}$ may impact the hemostatic balance of ACLD patients and may contribute to AICF. In the 1980s it was first observed that direct infusion of ascitic fluid into the circulation of ACLD patients (via LeVeen shunts) may lead to disseminated intravascular coagulation. ${ }^{9}$ Moreover, experimental studies showed that ascitic fluid has fibrinolytic properties. ${ }^{10,11}$ We hypothesized that ascitic fluid could have procoagulant properties and may contain extracellular vesicles that expose tissue factor (the main initiator of the blood coagulation cascade), because tissue factor exclusively associated with extracellular vesicles was detected in other body fluids including saliva, amniotic fluid, synovia, and seminal fluid. ${ }^{12-15}$

Importantly, ascitic fluid is reabsorbed into the blood circulation at high rates via the peritoneum. ${ }^{16,17}$ Reabsorption and peritoneal filtration of ascitic fluid are likely to change the composition of ascitic fluid, as the permeability of the peritoneum is inversely correlated with the molecular weight of ascitic fluid constituents (i.e., permeability decreases with increasing molecular weight). ${ }^{18}$ of note, the hemostatic properties of ascitic fluid that was membrane-filtered have yet to be investigated.

It was the aim of the present study to investigate the coagulation system in ascitic fluid and plasma of ACLD patients. We therefore included ACLD patients with ascites and ACLD patients without ascites, who were matched for the severity of portal hypertension (i.e., hepatic venous pressure gradient [HVPG]) and determined routine coagulation parameters and performed coagulation experiments. To mimic the function of the peritoneum as a semipermeable membrane, ${ }^{18}$ we also performed clotting and fibrinolysis experiments with ascitic fluid that was filtered through a $0.1-\mu \mathrm{m}$ membrane. Finally, we determined D-dimer (a biomarker that globally indicates coagulation activation and fibrinolysis) levels in a large thoroughly characterized cohort of patients with clinically significant portal hypertension (as defined by a HVPG $\geq 10 \mathrm{mmHg}$ ) to investigate whether coagulation activation and fibrinolysis are linked to ascites, independent of the severity of portal hypertension and/or hepatic dysfunction.

\section{Methods}

\section{Study Design and Population}

Cohort A (prospective study) consisted of adult patients with ACLD and clinically significant portal hypertension (i.e., HVPG $\geq 10 \mathrm{mmHg}$ ) and grade 3 ascites $^{19}$ who were scheduled for paracentesis, or similar patients without ascites, who were matched for HVPG. Patients with one or more of the following conditions were excluded: hepatocellular carcinoma (HCC) or other malignancies (active or in patients' history), history of transjugular intrahepatic portosystemic shunt (TIPS), liver transplantation or any other solid organ transplantation, kidney failure, as well as anticoagulation or venous/arterial thromboembolism, or antibiotic treatment or overt bacterial infection (including spontaneous bacterial peritonitis) within 3 months prior to screening. Ascitic fluid and blood were obtained from patients with ACLD and ascites who underwent clinically indicated paracentesis on the day of study inclusion. In patients with ACLD but without ascites (who did not undergo paracentesis), blood was collected on the day of study inclusion.

Cohort B (retrospective analysis) comprised ACLD patients without ascites or grade 1 to 3 ascites who underwent HVPG measurement at the Vienna Hepatic Hemodynamic Lab of the Medical University of Vienna between January 2006 and February 2018 and had clinically significant portal hypertension and available information on D-dimer levels. Patients with HCC or other malignancies, history of TIPS, or active bacterial infection were excluded.

Finally, we identified 14 patients in whom plasma Ddimer levels were determined before elective TIPS implantation for recurrent/refractory ascites and 1 to 3 months after the procedure within the Austrian registry on TIPS (AUTIPS, NCT03409263).

\section{Experimental and Routine Laboratory Assessments in Plasma (Cohorts A and B) and Ascitic Fluid (Cohort B) See the Supplementary Material (available in the online version).}

\section{HVPG Measurement in Cohorts A and B}

See the Supplementary Material (available in the online version).

\section{Statistical Analyses}

See the Supplementary Material (available in the online version).

\section{Ethics and Access}

See the Supplementary Material (available in the online version). 
Table 1 Characteristics of patients included in cohort A

\begin{tabular}{|c|c|c|c|c|}
\hline Patient characteristics & $\begin{array}{l}\text { All patients, } \\
n=50\end{array}$ & $\begin{array}{l}\text { Patients with grade } \\
3 \text { ascites, } \\
n=25\end{array}$ & $\begin{array}{l}\text { Patients without } \\
\text { ascites, } \\
n=25\end{array}$ & $\begin{array}{l}\text { p-Value } \\
\text { (patients with grade } \\
3 \text { ascites vs. } \\
\text { without ascites) }\end{array}$ \\
\hline Age, y & $57.8 \pm 11.9$ & $57.3 \pm 15$ & $58.3 \pm 8$ & 0.775 \\
\hline \multicolumn{5}{|l|}{ Sex } \\
\hline Male & $37(74 \%)$ & $4(16 \%)$ & $9(36 \%)$ & \multirow[t]{2}{*}{0.196} \\
\hline Female & $13(26 \%)$ & $21(84 \%)$ & $16(64 \%)$ & \\
\hline \multicolumn{5}{|l|}{ Etiology } \\
\hline Viral & $15(30 \%)$ & $5(20 \%)$ & $10(40 \%)$ & \multirow[t]{4}{*}{0.012} \\
\hline ALD & $22(44 \%)$ & $16(64 \%)$ & $6(24 \%)$ & \\
\hline NAFLD or cryptogenic & $10(20 \%)$ & $2(8 \%)$ & $8(32 \%)$ & \\
\hline Other & $3(6 \%)$ & $2(8 \%)$ & $1(4 \%)$ & \\
\hline Varices & $39(78 \%)$ & $20(80 \%)$ & $19(76 \%)$ & 0.733 \\
\hline History of variceal bleeding & $12(24 \%)$ & $5(20 \%)$ & $7(28 \%)$ & 0.508 \\
\hline Hepatic encephalopathy & $20(40 \%)$ & $17(68 \%)$ & $3(12 \%)$ & $<0.001$ \\
\hline HVPG, mmHg & $20.6 \pm 4.4$ & $21.4 \pm 4.8$ & $19.9 \pm 4$ & 0.229 \\
\hline MELD, points & $13(11-15)$ & $13(12-17)$ & $12(10-15)$ & 0.281 \\
\hline CTP score, points & $8(6-10)$ & $9(9-11)$ & $6(6-7)$ & $<0.001$ \\
\hline$A$ & $13(26 \%)$ & $0(0 \%)$ & $13(52 \%)$ & \multirow[t]{3}{*}{$<0.001$} \\
\hline B & $23(46 \%)$ & $13(52 \%)$ & $10(40 \%)$ & \\
\hline C & $14(28 \%)$ & $12(48 \%)$ & $2(8 \%)$ & \\
\hline Albumin, $g / L$ & $32.7 \pm 5.21$ & $30.9 \pm 4.1$ & $34.5 \pm 5.6$ & 0.013 \\
\hline Bilirubin, mg/dL & $1.47(0.99-2.5)$ & $2.02(1.09-3.45)$ & $1.41(0.94-2.12)$ & 0.281 \\
\hline INR & $1.4(0.2)$ & $1.4(1.3-1.5)$ & $1.5(1.25-1.65)$ & 0.281 \\
\hline Creatinine, mg/dL & $0.76(0.668-0.915)$ & $0.89(0.72-1.09)$ & $0.72(0.605-0.775)$ & 0.016 \\
\hline CRP, mg/L & $0.68(0.275-1.29)$ & $1.15(0.645-2.09)$ & $0.32(0.105-0.745)$ & 0.001 \\
\hline IL-6, $\mu \mathrm{g} / \mathrm{mL}$ & $14.8(7.8-36.7)$ & $29.9(20.1-46.4)$ & $8.02(3.57-13.3)$ & $<0.001$ \\
\hline $\mathrm{LBP}, \mu \mathrm{g} / \mathrm{mL}$ & $7.84 \pm 3.68$ & $9.31 \pm 3.48$ & $6.37 \pm 3.31$ & 0.004 \\
\hline
\end{tabular}

Abbreviations: ALD, alcoholic liver disease; CRP, C-reactive protein; CTP, Child-Turcotte-Pugh score; HVPG, hepatic venous pressure gradient; IL-6, interleukin 6; INR, international normalized ratio; LBP, lipopolysaccharide binding protein; MELD, model for end-stage liver disease; NAFLD, non-alcoholic fatty liver disease.

Note: Categorical variables are reported as numbers and proportions of patients, while continuous variables are shown as mean \pm standard deviation or median (interquartile range [IQR]), as appropriate.

All authors had access to the study data and reviewed and approved the final manuscript.

\section{Results}

Patient Characteristics According to the Presence or Absence of Ascites (Cohort A)

See - Table 1 and the Supplementary Material (available in the online version).

\section{Ascitic Fluid Is Procoagulant (Cohort A)}

To investigate the coagulant properties of ascitic fluid, we added ascitic fluid to platelet-free normal pooled plasma. Ascitic fluid was clearly procoagulant as it substantially shortened clotting times of platelet-free normal pooled plasma from a median of 2,083 seconds (interquartile range [IQR]: 1,732-2,696; addition of buffer) to a median of 592 seconds (IQR: 502-743; $p<0.001$ ) after addition of ascitic fluid (-Fig. 1).

Extracellular Vesicle-Associated Tissue Factor Activity Is High in Ascitic Fluid Compared with Plasma but Activities of Other Coagulation Factors Are Low or Nondetectable (Cohort A)

Next we investigated coagulation factor (F) activities in ascitic fluid and plasma ( - Fig. 2 and - Table 2). Extracellular vesicleassociated tissue factor activity was highly elevated in ascitic fluid compared with plasma of the same patients. Coagulation factor FII and FVII activities were low in ascitic fluid. FV, FVIII, and FX activities were not detectable in ascitic fluid. 


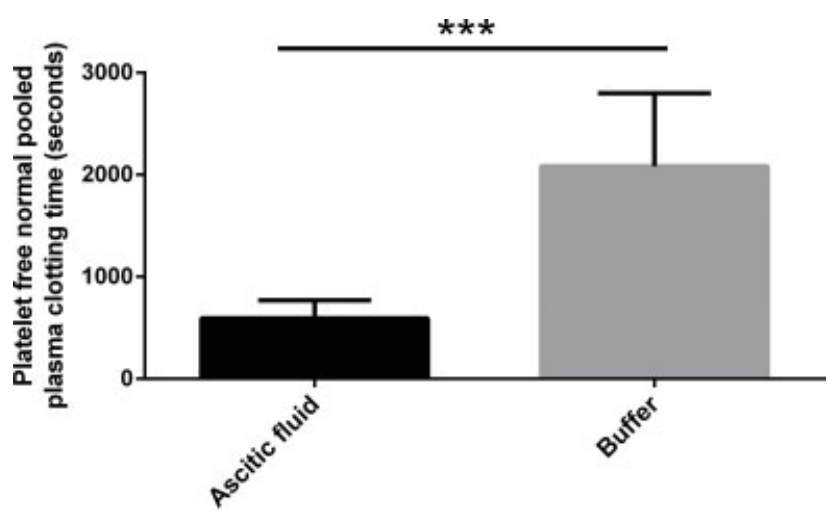

Fig. 1 Addition of ascitic fluid $(n=25)$ to platelet-free normal pooled plasma shortened the clotting time. ${ }^{* * *} p<0.001$. Data are presented as median with interquartile range and were analyzed by the MannWhitney $\mathrm{U}$ test.

\section{Tissue Factor-Exposing Extracellular Vesicles in Ascitic Fluid Induce Clotting (Cohort A)}

We added washed ascitic fluid-derived extracellular vesicles to vesicle-depleted normal pooled plasma, which shortened the clotting time from a median of $>7,200$ seconds (IQR: 3,992 to $>7,200$; addition of buffer) to a median of 1,418 seconds (IQR: 1,088-1,692; $p<0.001$; - Fig. 3). Ascitic fluid supernatant, which was depleted of extracellular vesicles, was not procoagulant and did not shorten the median clotting time ( $>7,200$ seconds [IQR: 4,802 to $>7,200]$ ) compared with buffer $(p=0.722)$. Also ascitic fluid that was filtered through a $0.1-\mu \mathrm{m}$ membrane (mimicking the peritoneal membrane) was not procoagulant (median clotting time: $>7200$ seconds [IQR: 0]) compared with buffer $(p=0.624)$. In another experiment, the procoagulant potential of ascitic fluid-derived extracellular vesicles was completely blocked by preincubation with a tissue factor-inhibiting antibody (median clotting time: $>7,200 \mathrm{sec}-$ onds [IQR: 2,434 to $>7,200$ ]; $p<0.001$ ).

Plasmin in Ascitic Fluid Induces Fibrinolysis (Cohort A) Incubation of generated fibrin clots in ascitic fluid for 24 hours resulted in partial clot lysis as evidenced by reduction in clot weight (-Fig. 4A) and D-dimer release in the supernatant ( $\mathbf{F i g}$. 4B). Specifically, we determined the change in D-dimer ( $\Delta$-D-dimer) after incubation of clots in ascitic fluid, in different ascitic fluid fractions and in normal saline. We found the same fibrinolytic potential in unprocessed ascitic fluid (median $\Delta$-D-dimer: 2,458 $\mu \mathrm{g} / \mathrm{mL}$ [IQR: 2,053-3,070]), extracellular vesicle-depleted ascitic fluid supernatant (median $\Delta$-D-dimer: $2,340 \mu \mathrm{g} / \mathrm{mL}$ [IQR: $1,894-3,035]$ ), and in ascitic fluid that was filtered through a $0.1-\mu \mathrm{m}$ membrane (median $\Delta$-D-dimer: 2,215 $\mu \mathrm{g} / \mathrm{mL}$ [IQR: 1,498-2,745]; $p=0.630$ ). In contrast, ascitic fluid-derived extracellular vesicles only had minimal fibrinolytic potential (median $\Delta$-D-dimer: $44 \mu \mathrm{g} / \mathrm{mL}$ [IQR: 5-571]; $p<0.001$; - Fig. 4B). Preincubation of ascitic fluid with aprotinin, which is an inhibitor of plasmin, completely blocked the fibrinolytic potential of ascitic fluid (median $\Delta$-D-dimer: $0.00 \mu \mathrm{g} / \mathrm{mL}$ [IQR: $0.00-1.84$ ]; $p<0.001$ ), with a median $\Delta$-D-dimer release comparable to clots suspended in normal saline (median $\Delta$-D-dimer: $12 \mu \mathrm{g} / \mathrm{mL}$ [IQR: 9-14]; - Fig. 4B).

\section{Markers of Coagulation Activation and Fibrinolysis Are Highly Elevated in Ascitic Fluid Compared with Plasma (Cohort A)}

Thrombin-antithrombin (TAT) complex, prothrombin fragment $1+2$, plasmin- $\alpha 2$-antiplasmin (PAP) complex, and Ddimer levels were highly elevated in ascitic fluid compared with plasma (-Table 2). Fibrinogen was not detectable in ascitic fluid suggesting the conversion of all fibrinogen into fibrin with subsequent degradation, as evidenced by the high D-dimer levels in ascitic fluid.

\section{Markers of Coagulation Activation and Fibrinolysis in Plasma of Patients with or without Ascites (Cohort A)}

We found low extracellular vesicle-associated tissue factor activity in plasma of ACLD patients with grade 3 ascites, which did not differ from extracellular vesicle-associated tissue factor activity in plasma of ACLD patients without ascites (-Table 2 ).
A

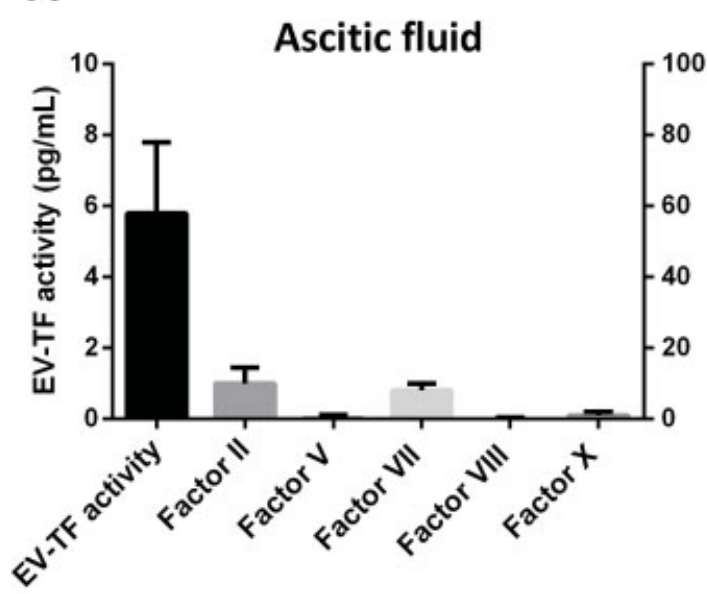

B

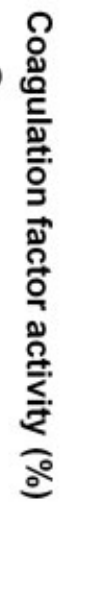

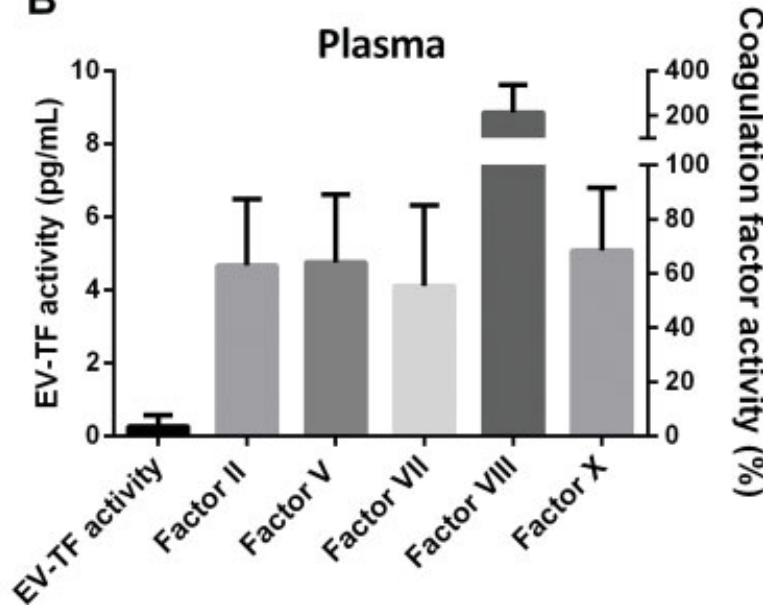

Fig. 2 Extracellular vesicle (EV)-associated tissue factor (TF) activity was high in ascitic fluid compared with plasma of the same patients ( $n=25$ ) (A). Activities of coagulation factors V, VIII, and X were not detectable in ascitic fluid and activities of coagulation factors II and VII were low in ascitic fluid compared with plasma (B). Data are presented as median with interquartile range. 
Table 2 Comparison of coagulation parameters between ascites and plasma of the same patients as well as plasma of patients with grade 3 ascites and plasma of patients without ascites in cohort $A$

\begin{tabular}{|c|c|c|c|c|c|}
\hline \multirow[t]{2}{*}{ Coagulation parameters } & \multicolumn{3}{|c|}{ Patients with grade 3 ascites, $n=25$} & \multirow{2}{*}{$\begin{array}{l}\text { Patients without ascites, } \\
n=25\end{array}$} & \multirow[b]{2}{*}{$p$-Value } \\
\hline & Ascitic fluid & Plasma & $p$-Value ${ }^{a}$ & & \\
\hline EV-TF activity, pg/mL & $5.79(4.78-8.01)$ & $0.18(0.00-0.72)$ & $<0.001$ & $0.15(0.08-0.29)$ & 0.946 \\
\hline FIl activity, \% & $9(5-14)$ & $59(44-65)$ & $<0.001$ & $54(48-63)$ & 0.662 \\
\hline FV activity, \% & $0(0-0)$ & $61(43-84)$ & $<0.001$ & $57(46-73)$ & 0.924 \\
\hline FVII activity, \% & $8(5-10)$ & $44(32-50)$ & $<0.001$ & $44(35-69)$ & 0.733 \\
\hline FVIII activity, \% & $0(0-0)$ & $237(210-292)$ & $<0.001$ & $176(149-218)$ & 0.027 \\
\hline FX activity, \% & $1(0-2)$ & $56(44-64)$ & $<0.001$ & $54(48-63)$ & 0.088 \\
\hline TAT complex, $\mu \mathrm{g} / \mathrm{mL}$ & $76.9(47.2-150.7)$ & $4.88(2.5-7.5)$ & $<0.001$ & $21.9(7.7-26.8 .1)$ & $<0.001$ \\
\hline $\begin{array}{l}\text { Prothrombin fragment } \\
1+2, \mathrm{pmol} / \mathrm{L}\end{array}$ & $1,836(956-2,796)$ & $174(126-262)$ & $<0.001$ & $285(171-349)$ & 0.018 \\
\hline PAP complex, $\mu \mathrm{g} / \mathrm{mL}$ & $10.9(7.9-14.7)$ & $0.9(0.7-1.1)$ & $<0.001$ & $0.3(0.2-0.5)$ & $<0.001$ \\
\hline D-dimer, $\mu \mathrm{g} / \mathrm{mL}$ & $68.0(37.8-102.5)$ & $5.20(3.8-7.4)$ & $<0.001$ & $0.7(0.5-1.7)$ & $<0.001$ \\
\hline$\alpha 2$-antiplasmin (\%) & $7(7-9)$ & $59(51-71)$ & $<0.001$ & $58(52-70)$ & 0.733 \\
\hline Plasminogen activity (\%) & $14(13-16)$ & $57(47-70)$ & $<0.001$ & $61(53-72)$ & 0.359 \\
\hline PAI-1 (IU/mL) & $0.28(0.0-1.14)$ & $0.0(0.0-1.6)$ & 0.948 & $0.28(0.0-2.25)$ & 0.583 \\
\hline Fibrinogen, mg/dL & $0(0-0)$ & $292(242-325)$ & $<0.001$ & $242(202-296)$ & 0.051 \\
\hline Peak TG, nM & - & $255(191-297)$ & - & $177(102-248)$ & 0.018 \\
\hline TG velocity index, $\mathrm{nM} / \mathrm{min}$ & - & $46.2(29.5-76.6)$ & - & $23.9(11.0-38.8)$ & 0.005 \\
\hline TG lag phase, min & - & $10.1(9.1-12.6)$ & - & $12.1(10.6-15.6)$ & 0.047 \\
\hline TG AUC/ETP, nM x min & - & $2,654(2,159-3,170)$ & - & $2,886(2,404-3,123)$ & 0.734 \\
\hline PT, \% & - & $57(51-66)$ & - & $58(57-69)$ & 0.397 \\
\hline APTT, s & - & $40(38-45)$ & - & $41(37-46)$ & 0.741 \\
\hline
\end{tabular}

Abbreviations: APTT, activated partial thromboplastin time; AUC, area under the curve; ETP, endogenous thrombin potential; EV-TF, extracellular vesicle-associated tissue factor activity; $F$, coagulation factor; PAI-1, plasminogen activator inhibitor-1; PAP, plasmin- $\alpha 2$-antiplasmin; PT, prothrombin time; TAT, thrombin-antithrombin; TG, thrombin generation.

Note: Data are reported as median (interquartile range [IQR]) and were analyzed by the Kruskal-Wallis test.

aplasma vs. ascitic fluid.

bPlasma of patients with grade 3 ascites vs. plasma of patients without ascites.

In plasma of patients with grade 3 ascites, peak thrombin generation (TG) and TG velocity index were increased, while TG lag phase was decreased compared with patients without ascites (-Table 2). In a multivariate analysis of factors associated with TG parameters, ascites was independently associated with increased peak TG (unstandardized regression coefficient $(B)$ : 50.2; $p=0.036 ;$-Supplementary Table S1, available in the online version). Accordingly, peak TG was $\sim 50 \mathrm{nM}$ higher in patients with ascites, even after adjusting for other patient characteristics. Moreover, there was a trend toward a decreased TG lag phase $(B:-3.04$; $p=0.083$; - Supplementary Table S2, available in the online version) in patients with ascites. In contrast, ascites was not independently linked to TG velocity index $(B: 9.67 ; p=0.281$; -Supplementary Table S3, available in the online version). Moreover, TAT complex and prothrombin fragment $1+2$ levels were lower in plasma of patients with ascites compared with patients without ascites (-Table 2); however, these differences disappeared after adjusting for other variables, and thus, ascites was not independently linked to these parameters $(B$ : $-3.73 ; p=0.645$; - Supplementary Table S4 (available in the online version), and $B$ : -100 ; $p=0.346$; - Supplementary Table S5, respectively).

Plasma D-dimer and PAP complex levels were elevated in patients with grade 3 ascites compared with patients without ascites (-Table 2 ) and ascites was an independent determinant of plasma D-dimer (B: 3.74; $p<0.001$; -Supplementary Table S6, available in the online version) and PAP complex levels ( $B: 443 ; p=0.002 ;$ - Supplementary Table S7, available in the online version) levels.

\section{Ascites Is Independently Linked to Coagulation Activation and Fibrinolysis in a cohort of 317 Patients with Advanced Chronic Liver Disease and Clinically Significant Portal Hypertension (Cohort B)}

Alcoholic liver disease was the most common etiology (42\%) and the median HVPG and model for end-stage liver disease (MELD) score were $19 \mathrm{mmHg}$ (IQR: 15-23) and 11 points 


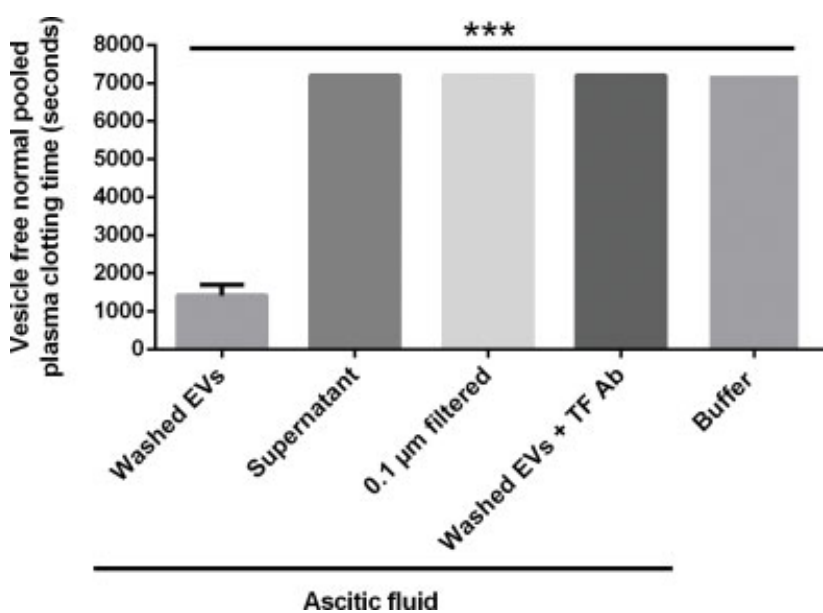

Fig. 3 Addition of ascitic fluid-derived washed extracellular vesicles (EVs) to vesicle-depleted normal pooled plasma shortened the clotting time $(n=25)$. Neither ascitic fluid supernatant nor membranefiltered ascitic fluid shortened the clotting time. Preincubation of ascitic fluid-derived EVs with a tissue factor antibody completely blocked the procoagulant potential of EVs; ${ }^{* * *} p<0.001$. Data are presented as median with interquartile range and were analyzed by the Kruskal-Wallis test.

(IQR: 9-14.5), respectively. Twenty-two percent of patients had Child-Turcotte-Pugh (CTP) score stage A, while 55 and $23 \%$ of patients were CTP stage B and C, respectively. Of note, due to similar inclusion criteria, there were no profound/clinically significant differences in patient characteristics between cohorts B and A (- Supplementary Table 58, available in the online version).

D-dimer levels showed a stepwise increase with ascites severity, as depicted in - Fig. 5. In a simple linear regression analysis, besides ascites severity, alcoholic liver disease, severity of portal hypertension (HVPG), and indicators of hepatic dysfunction (i.e., MELD and CTP score, hepatic

A

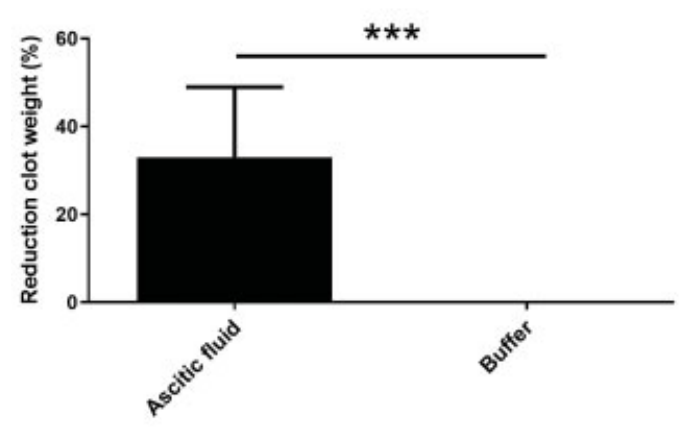

encephalopathy, as well as serum albumin and bilirubin levels) and systemic inflammation (C-reactive protein [CRP]) were linked to plasma D-dimer levels (-Table 3). Following backward elimination, ascites severity, HVPG $(p=$ 0.057), as well as serum albumin, bilirubin, and CRP levels were independently linked to plasma D-dimer levels. Accordingly, the association between ascites severity and D-dimer levels was independent of etiology, severity of portal hypertension and hepatic impairment, as well as systemic inflammation

\section{Changes in D-dimer Levels after TIPS for Recurrent/Refractory Ascites}

We stratified patients into those who clinically responded to TIPS (i.e., in whom ascites improved; $n=9$ ) and nonresponders $(n=5)$. There was a difference in the evolution of D-dimer levels ( $p=0.019)$ between those who responded to TIPS (i.e., in whom ascites improved; $n=9$; median $\Delta$-Ddimer: $-0.77 \mu \mathrm{g} / \mathrm{mL}$ [IQR: -0.35 to 2.46$]$ ]) and non-responders $(n=5$; median $\Delta$-D-dimer: $0.19 \mu \mathrm{g} / \mathrm{mL}$ [IQR: -3.15 to -0.16$])$ (-Supplementary Fig. S1, available in the online version).

\section{Discussion}

Ascitic fluid is a dynamic medium that enters and leaves the peritoneal cavity at high rates. ${ }^{16,20}$ In cohort $A$, we found that the coagulation system becomes activated intraperitoneally via tissue factor-exposing extracellular vesicles and generated fibrin gets lysed via plasmin generated within ascitic fluid. While tissue factor-exposing extracellular vesicles in ascitic fluid seem not to pass the peritoneal membrane and therefore do not enter the blood circulation in ACLD patients with ascites, our data indicate that hyperfibrinolysis in ascitic fluid (evidenced by strong fibrinolytic potential of ascitic fluid and high ascitic fluid PAP complex and D-dimer levels) could have systemic effects (evidenced by high PAP complex

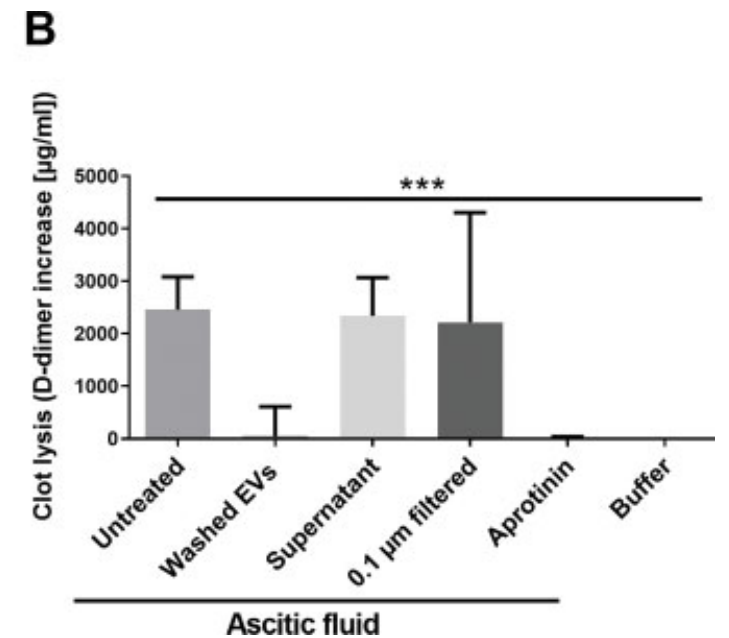

Fig. 4 (A) Incubation of fibrin clots in ascitic fluid $(n=25)$ for 24 hours reduced the weight of clots. (B) To further investigate the fibrinolytic potential of ascitic fluid, D-dimer increase ( $\Delta$-D-dimer) was determined after incubation of clots in ascitic fluid and different ascitic fluid fractions. High $\Delta$-D-dimer was found in untreated ascitic fluid and in ascitic fluid that was extracellular vesicle (EV)-depleted by centrifugation (supernatant) or filtration $(0.1 \mu \mathrm{m}$ filtered). Ascitic fluid-derived EVs had only minimal fibrinolytic potential. Preincubation of ascitic fluid with aprotinin, which is an inhibitor of plasmin, blocked the fibrinolytic potential of ascitic fluid. ${ }^{* * *} p<0.001$. Data are presented as median with interquartile range and were analyzed by the Kruskal-Wallis test. 
Table 3 Parameters associated with D-dimer levels in cohort B

\begin{tabular}{|l|l|l|l|l|l|l|}
\hline & A & B & B & C \\
\hline Patient characteristics & B & -Value & B & p-Value & B & $p$-Value \\
\hline Age, y & 0.01 & 0.527 & - & - & - & - \\
\hline Male sex & -0.161 & 0.686 & - & - & - & - \\
\hline ALD, vs. other etiologies & 1.42 & $<0.001$ & 0.106 & 0.743 & - & - \\
\hline Hepatic encephalopathy, CTP points & 1.55 & $<0.001$ & 0.016 & 0.946 & - & - \\
\hline HVPG, mmHg & 0.168 & $<0.001$ & 0.05 & 0.062 & 0.05 & 0.057 \\
\hline MELD, points & 0.273 & $<0.001$ & - & - & - & - \\
\hline CTP score, points & 0.76 & $<0.001$ & - & - & - & - \\
\hline Albumin, g/dL & -0.227 & $<0.001$ & -0.089 & 0.003 & -0.089 & 0.002 \\
\hline Bilirubin, mg/dL & 0.433 & $<0.001$ & 0.184 & 0.004 & 0.184 & 0.004 \\
\hline INR & 2.6 & $<0.001$ & - & - & - & - \\
\hline Creatinine, mg/dL & 2.56 & 0.958 & - & - & - & - \\
\hline CRP, mg/L & 2.76 & $<0.001$ & 1.9 & $<0.001$ & 1.92 & $<0.001$ \\
\hline Ascites, CTP points & 1.7 & $<0.001$ & 0.501 & 0.034 & 0.524 & 0.019 \\
\hline
\end{tabular}

Abbreviations: ALD, alcoholic liver disease; CRP, C-reactive protein; CTP, Child-Turcotte-Pugh score; HVPG, hepatic venous pressure gradient; INR, international normalized ratio.

Note: (A) Univariate analysis, (B) multivariate analysis, and (C) final step of backward selection of linear regression analysis.

and D-dimer levels in plasma). Consistently, in our second, large cohort of thoroughly characterized ACLD patients (cohort B), we found that D-dimer levels increase with ascites severity. The association between ascites severity and D-dimer levels was independent of liver disease etiology, severity of portal hypertension and hepatic impairment, as well as markers of systemic inflammation.

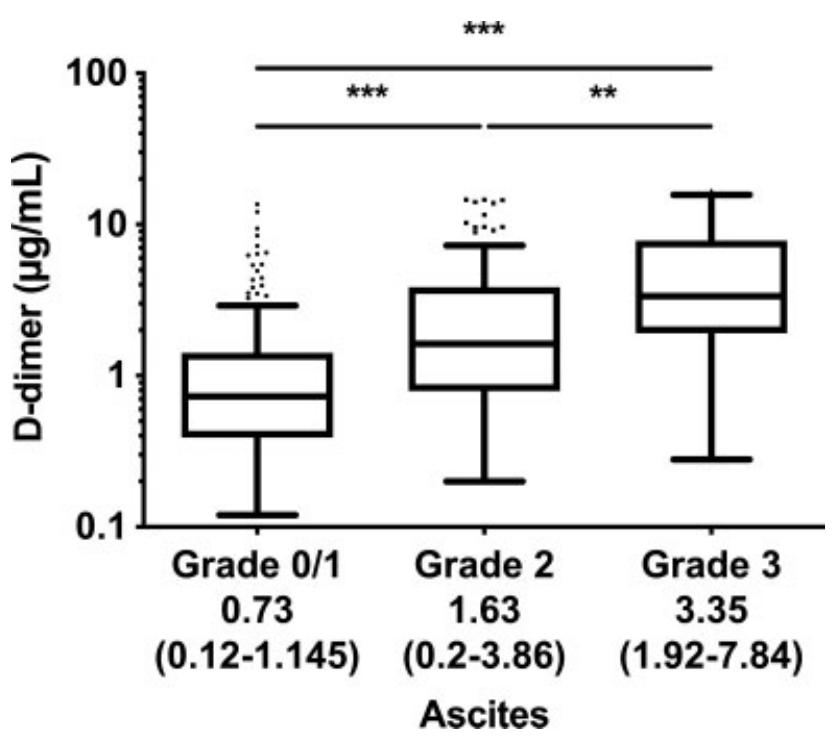

Fig. 5 In a large cohort (cohort $B ; n=317$ ) of advanced chronic liver disease patients with clinically significant portal hypertension (i.e., hepatic venous pressure gradient $\geq 10 \mathrm{mmHg}$ ), D-dimer levels increased with ascites severity. ${ }^{* *}$ Adjusted $p<0.05 .{ }^{* * *}$ Adjusted $p<0.001$. Data are presented as median with interquartile range and were analyzed by the Kruskal-Wallis test applying Dunn's multiple comparisons test.
Systemic hyperfibrinolysis in ACLD patients with ascites may have clinical significance, since it may contribute to nonportal hypertensive gastrointestinal bleeding events, which are common in ACLD patients and are associated with similarly high mortality, as compared with variceal bleeding. ${ }^{21}$ Current guidelines recommend the administration of vasoactive drugs and antibiotics as well as early endoscopy in patients with ACLD and upper gastrointestinal bleeding. ${ }^{19,22}$ Hyperfibrinolysis due to the presence of ascites may have a negative impact on the pharmacological/ endoscopic control of nonportal hypertensive bleeding, while an impact on portal-hypertensive gastrointestinal bleeding seems unlikely. Meta-analyses have shown that early administration of antifibrinolytic agents may reduce mortality in all-cause upper gastrointestinal bleeding ${ }^{23}$ and experts recommend the use of antifibrinolytic agents for delayed and diffuse mucosal bleeding in patients with decompensated ACLD. ${ }^{7}$ The recently published HALT-IT randomized controlled trial $^{24}$ failed to demonstrate a survival benefit in patients with gastrointestinal bleeding (including a considerable proportion of patients with suspected variceal bleeding or comorbid liver disease) who received tranexamic acid (which is an antifibrinolytic). However, no information on ascites was provided in the latter study or a previous meta-analysis. ${ }^{25}$ Of note, a randomized controlled trial investigating tranexamic acid in patients with ACLD and gastrointestinal bleeding is currently ongoing. ${ }^{6,26}$ Interestingly, D-dimer levels decreased to values comparable to those of patients without ascites after ascites resolution. ${ }^{27}$ Although D-dimer levels decreased both after paracentesis and diuretic treatment in another study, ${ }^{28}$ diuretic treatment achieved lower D-dimer levels that were even comparable to those of patients without ascites. This observation 
may be explained by small residual amounts of ascites after paracentesis or early reoccurrences of low volumes of ascites that are not always easily detectable. The results of these previous studies are well in line with our experimental and clinical findings and suggest that hyperfibrinolysis may also be ameliorated by ascites removal. Consistently, we found that improvement of ascites by TIPS placement impacted Ddimer levels in a series of patients from the Austrian registry on TIPS (AUTIPS, NCT03409263) who had paired D-dimer assessments before and 1 to 3 months after the procedure.

We detected low extracellular vesicle-associated tissue factor activity in plasma of ACLD patients with grade 3 ascites (cohort A). An explanation for the low extracellular vesicle-associated tissue factor activity in plasma of ACLD patients with ascites could be the relatively large size of tissue factor-exposing extracellular vesicles (above $0.1 \mu \mathrm{m}$ according to our investigations) for which the peritoneum seems to be largely impermeable under normal conditions. ${ }^{18,29}$ Another explanation could be clearance mechanisms in ascitic fluid/the peritoneal cavity that render the presence of tissue factor-exposing extracellular vesicles in plasma short-lived and therefore undetectable. However, tissue factor-exposing extracellular vesicles in ascitic fluid may impact the hemostatic balance of ACLD patients by generating fibrin, which is a cofactor of tissue-plasminogen activator. Fibrin increases the enzymatic activity of tissueplasminogen activator by several orders of magnitude, thereby strongly increasing plasmin generation in ascitic fluid and inducing its own fibrinolysis. ${ }^{30}$ In line, we detected substantially increased D-dimer and PAP complex levels in plasma of ACLD patients with ascites compared with patients without ascites and this association remained unaffected by adjustment for other factors, indicating an independent association. Consistent with our findings, an innovative translational study by Gitto et al detected highly elevated D-dimer levels as well as reduced plasminogen activity and plasminogen activator inhibitor-1 in ascitic fluid. ${ }^{31}$ Interestingly, Gitto et al also found reduced thrombin-activatable fibrinolysis inhibitor (TAFI) antigen levels but increased TAFI activity in ascitic fluid. Finally, findings from peritoneal dialysis patients who developed peritonitis suggest that the peritoneal permeability is increased under inflammatory conditions, ${ }^{32,33}$ and thus, the impermeability of the peritoneal membrane for extracellular vesicles needs to be reevaluated in patients with spontaneous bacterial peritonitis, who were excluded from our study.

Due to the relatively low number of patients in our prospective cross-sectional study (cohort A; $n=50$ ), we retrospectively investigated the association between D-dimer levels and ascites severity in a second cohort of 317 thoroughly characterized ACLD patients with clinically significant portal hypertension. D-dimer is a biomarker that globally reflects coagulation activation and fibrinolysis, because D-dimer is a plasmin degradation product of fibrin that was cross-linked by FXIII (at the very end of the coagulation cascade). In contrast to other biomarkers of coagulation activation and fibrinolysis, D-dimer has the unique advantage of being widely available in routine laboratories. Consistent with our experimental data, we found that D-dimer levels were independently associated with ascites severity in our second cohort, emphasizing the central role of ascites in ACLD-related hyperfibrinolysis. The exploratory mechanistical investigation of the procoagulant and fibrinolytic potential of ascitic fluid in a small but thoroughly characterized cohort of patients (cohort A) and the additional examination of the impact of ascites on plasma D-dimer levels in a larger independent cohort of patients (cohort B) may be regarded a strength of this study.

Besides increased fibrinolysis, we also detected an increased peak TG, TG velocity index, and a decreased TG lag phase in plasma of patients with ACLD and ascites, which may be interpreted as an increased "coagulation potential." This is in line with previous studies that reported increased endogenous thrombin potential in the presence of thrombomodulin in patients with CTP stages B and C, i.e., in subgroups with a high prevalence/severity of ascites. ${ }^{34}$ TAT complex and prothrombin fragment $1+2$ levels were lower in plasma of patients with ascites compared with patients without ascites. However, these differences lost statistical significance after adjusting for additional variables, indicating that ascites was not independently linked to these parameters.

It is a limitation of the present study that we did not investigate the cellular origin of tissue factor-exposing extracellular vesicles in ascitic fluid. We abstained from these investigations because it has been shown that the accuracy and reproducibility of antigen detection on extracellular vesicles is limited. ${ }^{35}$ However, evidence from previous studies that investigated tissue factor-exposing extracellular vesicles in other body fluids indicates that peritoneal epithelial cells are a likely source of tissue factor-exposing extracellular vesicles in ascitic fluid. ${ }^{12,14}$ Moreover, we did not investigate whether our observations also apply to other forms of ascites by including an additional group of patients without ACLD. Although the hemostatic equilibrium is nowadays considered to be balanced in most patients with ACLD, ACLD is still accompanied by substantial changes in hemostatic parameters that are related to the severity of liver disease. Thus, we abstained from studying patients without underlying ACLD, as they are inherently different, which substantially limits the significance of comparisons with such patients. The relatively low number of patients in cohort $A$ is another limitation of our study. Yet, these patients were prospectively recruited and matched for HVPG. Moreover, the link between ascites grade and fibrinolysis was confirmed in an independent large cohort of ACLD patients (cohort B); however, this part of our study was a retrospective analysis. Finally, our study did not investigate clinical endpoints related to hemostasis, i.e., bleeding or thrombosis.

In conclusion, we found that coagulation and fibrinolysis are activated intraperitoneally in patients with cirrhosis and ascites. While tissue factor-exposing extracellular vesicles in ascitic fluid seem unable to pass the peritoneal membrane, plasminogen gets activated into plasmin in ascitic fluid, may re-enter the circulation, and induce systemic hyperfibrinolysis. This may contribute to AICF, a common phenomenon in patients with decompensated cirrhosis, as well as nonportal 
hypertensive upper gastrointestinal bleeding events, which are associated with a high mortality.

\section{What is known about this topic?}

- The liver plays a central role in the hemostatic system since it synthesizes and clears most proteins involved in coagulation activation and fibrinolysis.

- Development of ascites is the most common form of decompensation of cirrhosis.

- Ascitic fluid is a dynamic medium that enters and leaves the peritoneal cavity at high rates.

\section{What does this paper add?}

- We found that coagulation and fibrinolysis are activated in ascites of patients with cirrhosis.

- While tissue factor-exposing extracellular vesicles in ascitic fluid seem unable to pass the peritoneal membrane, plasminogen gets activated into plasmin in ascitic fluid, may re-enter the circulation, and induce systemic hyperfibrinolysis.

- Consistently, D-dimer levels increase with ascites severity.

\section{Author Contributions}

J.T. and M.M. have contributed to the conceptualization, data curation, formal analysis, investigation, methodology, project administration, resources, supervision, validation, visualization, writing--original draft, as well as writing-review and editing, and J.T. additionally acquired funding specific to this study. T.L., P.Q., L.H., P.S., B.S., T.B., R.N., C.A., M.T., I.P., and T.R have contributed to data curation, investigation, methodology, resources, and writing-review and editing.

\section{Funding}

This work was supported by a grant from the Medical Scientific Fund of the Major of the City of Vienna (No. 15062), the Austrian Science Fund (FWF) Special Research Program SFB-F54, and by an unrestricted travel grant from the European Hematology Association (EHA).

\section{Conflict of Interest}

J.T and T.L. served as speakers and/or consultants and/or advisory board members for Daiichi Sankyo. P.Q. has served as a speaker and/or consultant and/or advisory board member for Roche and Takeda. P.S. received travel support from AbbVie, Falk, and Gilead. B.S. received travel support from AbbVie and Gilead. C.A. received honoraria for lectures and advisory boards from Bayer, Boehringer Ingelheim, Bristol-Myers Squibb, Daiichi Sankyo, and Pfizer. M.T. served as a speaker and/or consultant and/or advisory board member for Albireo, Boehringer Ingelheim, Bristol-Myers Squibb, Falk, Genfit, Gilead, Intercept, MSD, Novartis, Phenex, Regulus, and Shire, and received travel support from AbbVie, Falk, Gilead, and Intercept, as well as grants/research support from Albireo, Cymabay, Falk, Gilead, Intercept, MSD, and Takeda. He is also co-inventor of patents on the medical use of 24norursodeoxycholic acid. I.P. served as a speaker and/or consultant and/or advisory board member for Bayer, Boehringer Ingelheim, Bristol-Myers Squibb, Daiichi Sankyo, and Pfizer. T.R. served as a speaker and/or consultant and/or advisory board member for AbbVie, Bayer, Boehringer Ingelheim, Gilead, Intercept, MSD, Siemens, and W. L. Gore \& Associates and received grants/research support from AbbVie, Boehringer Ingelheim, Gilead, MSD, Philips, and W. L. Gore \& Associates as well as travel support from Boehringer Ingelheim and Gilead. M.M. served as a speaker and/or consultant and/or advisory board member for AbbVie, Bristol-Myers Squibb, Collective Acumen, Gilead, and W. L. Gore \& Associates, and received travel support from AbbVie, Bristol-Myers Squibb, and Gilead. L.H., T.B., and R.N. have nothing to disclose.

\section{References}

1 Tripodi A, Primignani M, Mannucci PM, Caldwell SH. Changing concepts of cirrhotic coagulopathy. Am J Gastroenterol 2017;112 (02):274-281

2 Lisman T, Porte RJ. Pathogenesis, prevention, and management of bleeding and thrombosis in patients with liver diseases. Res Pract Thromb Haemost 2017;1(02):150-161

3 Northup PG, Caldwell SH. Coagulation in liver disease: a guide for the clinician. Clin Gastroenterol Hepatol 2013;11(09):1064-1074

4 Lisman T, Bongers TN, Adelmeijer J, et al. Elevated levels of von Willebrand factor in cirrhosis support platelet adhesion despite reduced functional capacity. Hepatology 2006;44(01):53-61

5 Mandorfer M, Schwabl P, Paternostro R, et al; Vienna Hepatic Hemodynamic Lab. Von Willebrand factor indicates bacterial translocation, inflammation, and procoagulant imbalance and predicts complications independently of portal hypertension severity. Aliment Pharmacol Ther 2018;47(07):980-988

6 O'Leary JG, Greenberg CS, Patton HM, Caldwell SH. AGA clinical practice update: coagulation in cirrhosis. Gastroenterology 2019; 157(01):34.e1-43.e1

7 Intagliata NM, Argo CK, Stine JG, Lisman T, Caldwell SH, Violi Ffaculty of the 7th International Coagulation in Liver Disease. Concepts and controversies in haemostasis and thrombosis associated with liver disease: Proceedings of the 7th International Coagulation in Liver Disease Conference. Thromb Haemost 2018;118(08):1491-1506

8 D'Amico G, Pasta L, Morabito A, et al. Competing risks and prognostic stages of cirrhosis: a 25-year inception cohort study of 494 patients. Aliment Pharmacol Ther 2014;39(10):1180-1193

9 LeVeen HH, Ip M, Ahmed N, Hutto RB, LeVeen EG. Coagulopathy post peritoneovenous shunt. Ann Surg 1987;205(03):305-311

10 Agarwal S, Joyner KA Jr, Swaim MW. Ascites fluid as a possible origin for hyperfibrinolysis in advanced liver disease. Am J Gastroenterol 2000;95(11):3218-3224

11 Scott-Coombes DM, Whawell SA, Vipond MN, Crnojevic L, Thompson JN. Fibrinolytic activity of ascites caused by alcoholic cirrhosis and peritoneal malignancy. Gut 1993;34(08):1120-1122

12 Berckmans RJ, Sturk A, van Tienen LM, Schaap MC, Nieuwland R. Cell-derived vesicles exposing coagulant tissue factor in saliva. Blood 2011;117(11):3172-3180

13 Hell L, Wisgrill L, Ay C, et al. Procoagulant extracellular vesicles in amniotic fluid. Transl Res 2017;184:12-20

14 Berckmans RJ, Nieuwland R, Tak PP, et al. Cell-derived microparticles in synovial fluid from inflamed arthritic joints support 
coagulation exclusively via a factor VII-dependent mechanism. Arthritis Rheum 2002;46(11):2857-2866

15 Franz C, Böing AN, Hau CM, et al. Procoagulant tissue factorexposing vesicles in human seminal fluid. J Reprod Immunol 2013;98(1-2):45-51

16 Akay S, Ozutemiz O, Kilic M, et al. Reabsorption of ascites and the factors that affect this process in cirrhosis. Transl Res 2008;152 (04):157-164

17 Prentice TC, Siri W, Joiner EE. Quantitative studies of ascitic fluid circulation with tritium-labeled water. Am J Med 1952;13(06): 668-673

18 Flessner MF. Peritoneal transport physiology: insights from basic research. J Am Soc Nephrol 1991;2(02):122-135

19 European Association for the Study of the Liver. Electronic address: easloffice@easloffice.eu European Association for the Study of the Liver. EASL Clinical Practice Guidelines for the management of patients with decompensated cirrhosis. J Hepatol 2018;69(02):406-460

20 McKEE FW, Wilt WG Jr, et al. The circulation of ascitic fluid; interchange of plasma and ascitic fluid protein as studied by means of C14-labeled lysine in dogs with constriction of the vena cava. J Exp Med 1950;91(02):115-122

21 Ardevol A, Ibañez-Sanz G, Profitos J, et al. Survival of patients with cirrhosis and acute peptic ulcer bleeding compared with variceal bleeding using current first-line therapies. Hepatology 2018;67 (04):1458-1471

22 de Franchis R, Baveno VIFBaveno VI Faculty. Expanding consensus in portal hypertension: Report of the Baveno VI Consensus Workshop: stratifying risk and individualizing care for portal hypertension. J Hepatol 2015;63(03):743-752

23 Bennett C, Klingenberg SL, Langholz E, Gluud LL. Tranexamic acid for upper gastrointestinal bleeding. Cochrane Database Syst Rev 2014;2014(11):CD006640

24 Collaborators H-ITHALT-IT Trial Collaborators. Effects of a highdose 24-h infusion of tranexamic acid on death and thromboembolic events in patients with acute gastrointestinal bleeding (HALT-IT): an international randomised, double-blind, placebocontrolled trial. Lancet 2020;395(10241):1927-1936

25 Martí-Carvajal AJ, Solà I Antifibrinolytic amino acids for upper gastrointestinal bleeding in people with acute or chronic liver disease. Cochrane Database Syst Rev 2015;2015(06):CD006007
26 Heidet M, Amathieu R, Audureau E, et al. Efficacy and tolerance of early administration of tranexamic acid in patients with cirrhosis presenting with acute upper gastrointestinal bleeding: a study protocol for a multicentre, randomised, double-blind, placebocontrolled trial (the EXARHOSE study). BMJ Open 2018;8(08): e021943

27 Spadaro A, Tortorella V, Morace C, et al. High circulating D-dimers are associated with ascites and hepatocellular carcinoma in liver cirrhosis. World J Gastroenterol 2008;14(10):1549-1552

28 Saray A, Mesihovic R, Gornjakovic S, et al. Association between high D-dimer plasma levels and ascites in patients with liver cirrhosis. Med Arh 2012;66(06):372-374

29 Henderson JM, Stein SF, Kutner M, Wiles MB, Ansley JD, Rudman D. Analysis of Twenty-three plasma proteins in ascites. The depletion of fibrinogen and plasminogen. Ann Surg 1980;192 (06):738-742

30 Medved L, Nieuwenhuizen W. Molecular mechanisms of initiation of fibrinolysis by fibrin. Thromb Haemost 2003;89(03): 409-419

31 Gitto S, Romanelli RG, Cellai AP, et al. Altered clot formation and lysis are associated with increased fibrinolytic activity in ascites in patients with advanced cirrhosis. Intern Emerg Med 2021;16 (02):339-347

32 Zemel D, Koomen GC, Hart AA, ten Berge IJ, Struijk DG, Krediet RT. Relationship of TNF-alpha, interleukin-6, and prostaglandins to peritoneal permeability for macromolecules during longitudinal follow-up of peritonitis in continuous ambulatory peritoneal dialysis. J Lab Clin Med 1993;122(06):686-696

33 Zemel D, Betjes MG, Dinkla C, Struijk DG, Krediet RT. Analysis of inflammatory mediators and peritoneal permeability to macromolecules shortly before the onset of overt peritonitis in patients treated with CAPD. Perit Dial Int 1995;15(02): 134-141

34 Tripodi A, Salerno F, Chantarangkul V, et al. Evidence of normal thrombin generation in cirrhosis despite abnormal conventional coagulation tests. Hepatology 2005;41(03):553-558

35 van der Pol E, Sturk A, van Leeuwen T, Nieuwland R, Coumans FISTH-SSC-VB Working group. Standardization of extracellular vesicle measurements by flow cytometry through vesicle diameter approximation. J Thromb Haemost 2018;16(06): $1236-1245$ 